\title{
The Prognostic Role of Lactate Dehydrogenase at First Relapse of Multiple Myeloma
}

\author{
Holly Lee ${ }^{a}$ Victor H. Jimenez-Zepeda ${ }^{a, b}$ \\ a Division of Hematology, Department of Medicine, Tom Baker Cancer Center, University of \\ Calgary, Calgary, AB, Canada; ${ }^{\mathrm{b}}$ Arnie Charbonneau Cancer Institute, Calgary, AB, Canada
}

\section{Editorial comment on the paper by Liu et al. Serum Lactate Dehydrogenase Can Be Used as a Factor for Re-Evaluating First-Relapsed Multiple Myeloma. An Original Paper. Acta Haematol 2020;143:559-566}

In this issue of Acta Haematologica, Liu et al. [1] discuss the prognostic impact of lactate dehydrogenase (LDH) at first relapse of multiple myeloma patients.

Elevated LDH is a marker of poor prognosis at the time of diagnosis of multiple myeloma. Increased LDH is associated with worse overall survival, progression-free survival (PFS), aggressive disease, and high tumor burden [2-4]. The Revised International Scoring System (R-ISS) for myeloma, which incorporates high $\mathrm{LDH}$, high beta-2 microglobulin, low albumin, and the presence of highrisk chromosomal abnormalities [4], is an effective clinical tool to evaluate the risk and prognosis of patients with newly diagnosed multiple myeloma.

Despite the rapidly changing therapeutic landscape of multiple myeloma with the success of novel agents, the disease remains incurable, and most patients experience relapse. Based on the recommendations from International Myeloma Working Group (IMWG), there are a number of factors at relapse that predict poor patient outcome, including the presence of primary refractory disease, relapse within 1 year from autologous stem cell transplant, progression within the first year of diagnosis, high-risk fluorescence in situ hybridization cytogenetic features including del(17p), $\mathrm{t}(4 ; 14), \mathrm{t}(14 ; 16), \mathrm{t}(14 ; 20)$, amp (1q21), ISS stage at relapse, and high-risk gene expression profile [5].

There is limited data on the significance of LDH measured at relapse and its role in disease re-staging and prognosis [2]. The clinical implications of high LDH at relapse has been reported previously in a prospective study of 99 patients with relapsed or refractory multiple myeloma treated with lenalidomide and dexamethasone with or without bortezomib. The study included patients who received a median of two previous lines of therapy ( $48 \%$ of the patients received three or more lines of previous treatment). In this study population, $\mathrm{LDH}>300 \mathrm{U} / \mathrm{L}$ at relapse was associated with inferior response to therapy and shorter PFS [2].

In order to further investigate the role of $\mathrm{LDH}$, specifically at first relapse, Liu et al. [1] conducted a retrospective study of 112 multiple myeloma patients who experienced first-time relapse. Initial induction treatment was heterogeneous with more than half of the patients receiving cyclophosphamide, bortezomib, and dexamethasone (CyBorD). Seven patients underwent autologous hematopoietic stem cell transplantation. Based on the risk stratification by ISS and Durie Salmon staging, high-risk patients received bortezomib or lenalidomide

karger@karger.com
www.karger.com/aha
Karger

Victor H. Jimenez-Zepeda, MD

Tom Baker Cancer Center, University of Calgary 133129 th St, NW

Calgary, AB T2N 4N2 (Canada)

victor.zepeda@albertahealthservices.ca 
maintenance, and low-risk patients received thalidomide maintenance. LDH was measured at first relapse, and patients were categorized to high-LDH and normal-LDH groups based on a LDH cutoff of $240 \mathrm{U} / \mathrm{L}$.

The study highlighted that elevated $\mathrm{LDH}$ at relapse was associated with higher beta 2 microglobulin, lower albumin, higher rates of extramedullary disease, presence of 1q21 amplification, and a trend towards an increased rate of del(17p). The high-LDH group had lower rates of response to salvage treatment with $35.1 \%$ achieving $\geq$ partial response, and $5.4 \%$ achieving complete response, compared to $82.1 \% \geq$ partial response and $21.4 \%$ complete response rates in the normal-LDH group. High LDH and ISS stage $2 / 3$ at relapse were significant prognostic factors of overall survival. Both elevated LDH and response to salvage treatment were significant predictors of PFS2, which in this study was defined as the time from first relapse to second progression. Notably, 40 of the 112 patients (35.7\%) had high LDH at relapse, which was considerably higher than the reported $12 \%$ of the patients with high LDH at diagnosis in the previous R-ISS publication cohort [4].

The study is limited given its retrospective design and small sample size. Data on fluorescence in situ hybridization chromosomal analysis on purified plasma cells was available in only 41 patients (36.6\%) and thus not included in the multivariate analysis. Initial patient risk stratification was performed using ISS and Durie Salmon staging. Despite these limitations, the study provides valuable insight into the prognostic role of LDH and its importance in restaging patients with multiple myeloma at first relapse. LDH is an easily accessible and relatively inexpensive clinical biomarker. Prospective studies with a larger patient population and risk stratification based on R-ISS are required to further validate the results of this study.

\section{Disclosure Statement}

The authors do not have conflicts of interest to disclose.

\section{Funding Sources}

There are no funding sources to report.

\section{Author Contributions}

H.L. and V.H. J.-Z. wrote the manuscript.

\section{References}

1 Liu Y, Wen L, Chen H, Chen Y, Duan W, Kang Y, et al. Serum lactate dehydrogenase can be used as a factor for re-evaluating first relapsed multiple myeloma. Acta Haematol. DOI: $10.1159 / 000505737$.

2 Dimopoulos MA, Kastritis E, Christoulas D, Migkou M, Gavriatopoulou M, Gkotzamanidou M, et al. Treatment of patients with relapsed/refractory multiple myeloma with lenalidomide and dexamethasone with or without bortezomib: prospective evaluation of the impact of cytogenetic abnormalities and of previous therapies. Leukemia. 2010 Oct; 24(10):1769-78.
3 Terpos E, Katodritou E, Roussou M, Pouli A, Michalis E, Delimpasi S, et al.; Greek Myeloma Study Group, Greece. High serum lactate dehydrogenase adds prognostic value to the international myeloma staging system even in the era of novel agents. Eur J Haematol. 2010 Aug;85(2):114-9.
4 Palumbo A, Avet-Loiseau H, Oliva S, Lokhorst HM, Goldschmidt H, Rosinol L, et al. Revised International Staging System for Multiple Myeloma: A Report From International Myeloma Working Group. J Clin Oncol. 2015 Sep;33(26):2863-9.

5 Laubach J, Garderet L, Mahindra A, Gahrton G, Caers J, Sezer O, et al. Management of relapsed multiple myeloma: recommendations of the International Myeloma Working Group. Leukemia. 2016 May;30(5):1005-17. 